\title{
In Praise of a Cumulative Prevention Science ${ }^{1}$
}

\author{
Richard H. Price ${ }^{2}$
}

University of Michigan

Durlak and Wells (1997) provide a pivotal appraisal of prevention research on children and adolescents. Their meta-analytic approach has the advantages of reducing scientific misjudgments based on single studies, and providing a more balanced evaluation of impact of various interventions; it provides an opportunity for hypothesis finding, helps set methodological standards, allows assessment of working classifications in the field, and an evaluation of the maturity of the prevention field itself. New developmental tasks for the field include incorporating and pursuing the leads produced by these findings, conducting similar research syntheses with other populations and outcomes, and using the results as an impetus to increased operational precision and parsimony.

KEY WORDS: prevention research; meta-analytic approach.

The Structure of Scientific Revolutions (Kuhn, 1962) is one of the most influential works in the philosophy of science in the last half century. Kuhn argued that normal science is usually a cumulative stream of activity dominated by a single paradigm, but occasionally this stream is punctuated by revolutionary upheavals. In these upheavals the normal paradigm is overthrown by a new intellectual order. While Kuhn meant his work to refer exclusively to the physical sciences, social and behavioral scientists heard his general thesis as a clarion call. For many social scientists in different theoretical camps it was attractive to believe they possessed the revolutionary paradigm among many competing paradigms (Price, 1978).

1Work on this paper was supported by National Institute of Mental Health Grant 5P30MH38330 to the Michigan Prevention Research Center.

${ }^{2}$ All correspondence should be addressed to Richard H. Price, Institute for Social Research, The University of Michigan, P.O. Box 1248, Ann Arbor, Michigan 48106-1248.

169

0091-0562/97/0400-0169\$12.50/0 \& 1997 Plenum Publishing Corporation 
As a result, they found the activities of cumulative normal science much less compelling than making claims for their own favorite paradigm. The social and behavioral sciences have been much poorer as a result.

While one wing of the social sciences is currently moving away from positivism itself, and toward interpretation for its own sake (Gergen, 1994), there are encouraging signs that a cumulative behavioral and social science is beginning to develop in other quarters (Lipsey \& Wilson, 1993). One of the principal vehicles for this new emphasis on cumulativeness is metaanalysis (Hunter, Schmidt, \& Jackson, 1982; Rosenthal, 1991). Metaanalysis has allowed the recent summary and appraisal of numerous fields of research. The paper by Durlak and Wells (1997) promises to establish a similar beachhead for prevention research.

Even though the accomplishments are impressive, there are still those who remain uninformed or skeptical about the potential contributions of meta-analysis as an addition to the methodological repertoire of the social sciences. While skepticism is a virtue among scientists, it sometimes reflects adherence to older traditions that have served us less well than we imagined. One of these is an uncritical reliance on statistical significance tests (Schmidt, 1996) and another is the well-documented tendency among humans, and even scientists, to be influenced by judgment biases (Kahneman, 1982; Tversky, 1988). For example, we are likely to be inclined to favor one vivid and concrete example of a single research finding over better evidence that takes into account a whole distribution findings, repeatedly sampled from a population of studies.

\section{A PIVOTAL APPRAISAL}

The Durlak and Wells (1997) paper is a powerful example of how a meta-analysis can, at the right moment in a maturing field, provide a pivotal appraisal and point out new opportunities for further high impact research. Among the advantages illustrated by the Durlak and Wells meta-analysis are that (a) it provides a counterweight to misjudgments based on findings from a single study, (b) it can help identify high potency interventions and allow systematic evaluation of other interventions whose effectiveness may have been overestimated, (c) it can aid in finding promising new hypotheses, (d) it can provide an empirical basis for identifying methodological best practices, (e) it can help in evaluating the plausibility of a working classification system for a growing field, and finally, (f) it provides a crucial supplement to individual expert opinion in evaluating the promise and maturity of a new field such as prevention research. 


\section{Scientific Misjudgments}

Schmidt and his colleagues (Hunter et al. 1982; Schmidt \& Hunter, 1992, 1996) have demonstrated in a variety of studies and analytical reports that social and behavioral scientists systematically misunderstand and overvalue statistical significance tests in interpreting data and dramatically underestimate the role of measurement error in evaluating the contribution of any single study. The Durlak and Wells (1997) results show that, even among studies of a particular intervention or particular target group, effect sizes can vary widely. Clearly, a focus on results of any single study can lead to substantial overestimations or underestimations of impact for a particular intervention. Breakthrough studies, no matter how often celebrated, are unusual and a sober assessment of the promise of any preventive intervention must await an appraisal of the cumulative evidence.

\section{Evaluating the Impact of Interventions}

Examined in isolation, the impact of any particular preventive intervention strategy can be evaluated only in comparison with outcomes in its comparison or control group. Preventive interventions that demonstrate their potency across a range of populations and circumstances, on the other hand, suggest a robustness that is to be desired, both for pragmatic reasons, and because an unpacking of their critical ingredients can be theoretically informative. Similarly, discovering that the effect sizes of an intervention that has been strongly endorsed are not what had been hoped can lead to appropriate revisions practice. For example, the somewhat surprising lack of impact of parent training strategies may call for an evaluation of the strategies and tactics of program recruitment and implementation rather than an abandonment of the approach.

\section{Hypothesis Finding}

New research leads can be stimulated by meta-analysis. When a metaanalysis reveals that the impact of a preventive intervention is moderated by some other parameter such as age or developmental period, the circumstances are ripe for formulating testable hypotheses that can advance the field. For example, consider the finding reported by Durlak and Wells (1997) that both affective education and problem-solving techniques had reliable impacts on younger rather than older children. That age or developmental stage moderates the effect of these interventions calls out for developmental hypotheses that view the intervention as a developmental influence may interact with unfolding developmental capacities in the child. 


\section{Setting Methodological Standards}

Summarizing the typical measurement and design practices in a meta-analysis confers additional advantages. Practices such as the prevalence of collection of follow-up data can be evaluated, as Durlak and Wells (1997) have done, and recommendations can be made when designs fall short of prevailing standards or preclude conclusions that would advance the field. Clearly in the case of the present summary by Durlak and Wells, a $25 \%$ rate of follow-up data collection is suboptimal and could be easily improved in the next generation of prevention research. Based on their analysis, Durlak and Wells make a number of other recommendations that could help set scientific standards for editorial boards and funding agencies seeking to improve the state of the art in prevention research. They rightly mention, for example, the need for more precise operational definitions, detailed program documentation, and evidence of implementation. Beyond that, methodological research using meta-analytic techniques can be used to evaluate the consequences of various methodological practices as Heinsman and Shadish (1996) have shown in a convincing piece of research on the relative merits of combining assignment methods in research designs.

\section{Assessing the Plausibility of a Working Classification}

Prevention researchers have engaged in a prolonged and sometimes heated debate about the value of various ways of characterizing their research and intervention efforts. These debates have seldom had the benefit of empirical data. Instead, they have relied on a priori statements of preference for promotion versus prevention strategies and environmental versus person centered approaches to intervention. Meta-analysis techniques allow an assessment of the plausibility of such distinctions, and also allow some systematically summarized data to be brought to bear against claims of superiority of one approach versus another. For example, the current study by Durlak and Wells seems to indicate that promotion and prevention strategies have roughly equal impacts of the outcomes examined in this review.

While this use of meta-analytic techniques is helpful, it is worth noting the assumption underlying the evaluation of categories and classifications of prevention strategies used by Durlak and Wells. Meta-analysis and related modeling techniques for research synthesis (Hcdges \& Olkin, 1985) can assess the usefulness of existing classifications, but only in the sense that it is possible to evaluate homogeneity of outcomes according to alterative classification 
schemes. That is, categories are deemed plausible to the degree that the intracategory variance in outcomes is smaller than alternative possible classifications. This is a useful and important criterion, but sets aside the question of whether there are other unmeasured sources of intracategory heterogeneity such as sample characteristic differences or outcome measurement differences that influence variation in outcomes within categories.

\section{Assessing the Maturity of the Field of Prevention Research}

Meta-analysis can provide the opportunity to ask, how is this field doing? Is it characterized by a few encouraging findings, or are repeated and converging findings stimulating new research directions and increasing confidence in the reliability and impact of preventive interventions? These questions are of great interest not only to researchers in the field but also to funders, policy makers, and practitioners, all of whom are interested in reliable summary information (Haggerty \& Mrazek, 1994). Policy makers might be particularly interested in how well a prevention strategy compares in its impact with treatment approaches that represent alternative policy or practice choices (Kazdin, 1990). For example, measures of relative impact such as effect sizes for interventions in conjunction with benefit-cost analyses (Gramlich, 1990) can signal which types of interventions might represent wise policy investments.

\section{A CRITICAL PERIOD FOR PREVENTION RESEARCH}

Just as it is plausible that there are critical periods or turning points in the lives of individuals, it is also reasonable to consider the possibility that similar turning points exist for entire fields of research. The current meta-analysis by Durlak and Wells (1997) may signal such a turning point in the prevention field. Like all turning points, the field of prevention research is presented both with new opportunities and new and critical developmental tasks. If our competencies are equal to the tasks, the field will experience a new maturity and viability. What then, might some of those developmental tasks be? 
Taking the Durlak and Wells

Findings Seriously

Systematic reviews of this kind unearth findings that were not previously a part of the collective consciousness of prevention researchers. For them to become part of the collective consciousness of the prevention research field requires both recognition of the issues uncovered and debate about their merits. For example, what are we to make of the finding reported by Durlak and Wells (1997) that most interventions, regardless of their claims as prevention or promotion, influenced both competence and psychosocial problems? Many researchers claim a more or less exclusive focus on either prevention or promotion approaches, believing that they are either promoting competence or preventing problems. Perhaps it is time to reflect on the unifying developmental models underlying our preventive efforts. Such unifying models will almost certainly point to reciprocal causal influences between the development of competence and problems of adaptation. Or consider another example. It appears that in the studies examined by Durlak and Wells, the effect sizes of behavioral approaches are twice the magnitude of nonbehavioral approaches. Can we unpack and compare these two methods to identify operational differences between behavioral and nonbehavioral approaches that will lead to both a more powerful and more conceptually sophisticated intervention technology?

\section{More Meta-Analyses Needed}

A second developmental task might be to supplement the initial gains made by the Durlak and Wells (1997) review with still others. For example, the present review confined itself to young children and adolescents, leaving the rest of the life course unexamined. Other critical transitions such as from school to work, entering and leaving roles in marriage, work, parenthood, caregiving, and illness deserve equal attention and may yield similar benefits. In addition, meta-analyses conducted in related fields (Lipsey \& Wilson, 1993; Slavin, Karweit, \& Wasik, 1994; Tobler, 1992) reinforce the prevention message and allow comparison across diverse outcomes and fields of study. For example, it would be surprising if the tactics of behavior change used in educational interventions, drug abuse prevention studies, and workplace intervention research, considered together did not display uniformities that were informative for future research. 


\section{Sharpening Definitions and Using Occam's Razor}

Reviews such as this are only as good as the definitions, both theoretical and operational, that have been previously used in the field, or that can be creatively synthesized by the reviewers. A developmental task high on the prevention research agenda is an insistence on more and better operational definitions, and more precision and clarity in their formulation. It is possible and even likely that some cherished distinctions, such as that between person and environment, and between promotion and prevention goals will blur and others may become clearer when more precise operational definitions and Occam's criterion of parsimony are conscientiously taken on as developmental tasks for the field.

\section{REFERENCES}

Durlak, J. A., \& Wells, A. M. (1997). Primary prevention mental health programs for children and adolescents: A meta-analytic review. American Joumal of Community Psychology, 25, 115-152.

Gergen, K. J. (1994). Realities and relationships: Sounding in social construction. Cambridge, MA: Harvard University Press.

Gramlich, E. M. (1990). A guide to benefit-cost analysis. Englewood Cliffs, NJ: Prentice Hall.

Haggerty, R. J., \& Mrazek, P. J. (1994). Reducing risks for mental disorders: Frontiers for preventive intervention research. Washington, DC: National Academy Press.

Hedges, L. V., \& Olkin, I. (1980). Statistical methods for meta-analysis. Orlando, FL: Academic Press.

Heinsman, D. T., \& Shadish, W. R. (1996). Assignment methods in experimentation: When do nonrandomized experiments approximate answers from randomized experiments? Psychological Methods, 1, 154-169.

Hunter, J. E., Schmidt, F. L., \& Jackson, G. B. (1982). Meta-analysis: Cumulating research findings across studies. Beverly Hills, CA: Sage.

Kahneman, D. (1982). Judgment under uncerzainty: Heuristics and biases. New York: Cambridge University Press.

Kazdin, A. E. (1990). Psychotherapy for children and adolescents. Annual Review of Psychology, 41, 21-54.

Kuhn, T. S. (1962). The structure of scientific revolutions. Chicago: University of Chicago Press.

Lipsey, M. W., \& Wilson, D. B. (1993). The efficacy of psychological, educational and behavioral treatment: Confirmation from meta-analysis. American Psychologist, 48, 1181-1209.

Price, R. H. (1978). Abnomal behavior. Perspectives in conflict (2nd ed.). New York: Holt, Rinehart and Winston.

Rosenthal, R. (1991). Meta-analytic procedures for social research (2nd ed.). Newbury Park, CA: Sage.

Schmidt, F. L. (1996). Statistical significance testing and cumulative knowledge in psychology: Implications for training of researchers. Psychological Methods, 1, 115-129.

Schmidt, F. L., \& Hunter, J. E. (1992). Moderator research and the law of small numbers. Pessonnel Psychology, 47, 1173-1181.

Schmidt, F. L., \& Hunter, J. E. (1996). Measurement error in psychological research: Lessons from 26 research scenarios. Psychological Methods, 1, 199-223. 
Slavin, R. E., Karweit, N. L., \& Wasik, B. A. (1994). Preventing early school failure. Needham Heights, MA: Allyn \& Bacon.

Tobler, N. S. (1992). Drug prevention programs can work: Research findings. Journal of Addictive Diseases, 11, 1-28.

Tversky, A. (1988). Decision making: Descriptive, normative, and prescriptive interactions. New York: Cambridge University Press. 